\title{
Lymph Node Thyroglobulin Measurement in Diagnosis of Neck Metastases of Differentiated Thyroid Carcinoma
}

\author{
Luca Giovanella, ${ }^{1,2}$ Luca Ceriani, ${ }^{1}$ and Sergio Suriano ${ }^{1}$ \\ ${ }^{1}$ Department of Nuclear Medicine and Thyroid Unit, Oncology Institute of Southern Switzerland, Street Ospedale 12, \\ 6500 Bellinzona, Switzerland \\ ${ }^{2}$ Department of Clinical Chemistry and Laboratory Medicine, Ente Ospedaliero Cantonale, 6500 Bellinzona, Switzerland
}

Correspondence should be addressed to Luca Giovanella, luca.giovanella@eoc.ch

Received 27 January 2011; Revised 16 March 2011; Accepted 30 March 2011

Academic Editor: Electron Kebebew

Copyright (C) 2011 Luca Giovanella et al. This is an open access article distributed under the Creative Commons Attribution License, which permits unrestricted use, distribution, and reproduction in any medium, provided the original work is properly cited.

\begin{abstract}
Aim. Enlarged cervical lymph nodes (LNs) in patients with thyroid cancer are usually assessed by fine-needle aspiration cytology (FNAC). Thyroglobulin (Tg) is frequently elevated in malignant FNAC needle wash specimens (FNAC-Tg). The objectives of the study were to (1) determine an appropriate diagnostic cut-off for FNAC-Tg levels (2) compare FNAC and FNAC-Tg results in a group of 108 patients affected by differentiated thyroid carcinoma (DTC). Methods. A total of 126 consecutive FNACs were performed on enlarged LNs and the final diagnosis was confirmed by surgical pathology examination or clinical follow-up. The best FNAC-Tg cut-off level was selected by receiver operating curve analysis, and diagnostic performances of FNAC and FNAC-Tg were compared. Results. The rate of FNAC samples adequate for cytological examination was 77\% in contrast FNAC-Tg available in $100 \%$ of aspirates $(P<.01)$. The sensitivity, specificity, and accuracy of FNAC were $71 \%, 80 \%, 74 \%, 100 \%, 80 \%$, and $94 \%$, respectively. The most appropriate cut-off value for the diagnosis of thyroid cancer metastatic LN was $1.1 \mathrm{ng} / \mathrm{mL}$ (sensitivity $100 \%$, specificity 100\%). Conclusions. The diagnostic performance of needle washout FNAC-Tg measurement with a cut-off of $1.1 \mathrm{ng} / \mathrm{mL}$ compared favorably with cytology in detecting DTC node metastases.
\end{abstract}

\section{Introduction}

The prognosis of patients who receive appropriate treatment for thyroid carcinoma is usually favourable, especially for differentiated thyroid carcinoma (DTC). However, although most patients have a long-term survival rate, $5 \%$ to $20 \%$ of patients will develop recurrence during the followup, primarily in the cervical lymph nodes (LNs) $[1,2]$. These LNs metastases may be detected clinically, but now are most often discovered on ultrasonography (US) [3]. It is of great importance to differentiate accurately LN metastases from benign reactive lymph nodes in order to avoid unnecessary treatment, but also to treat metastatic patients without delay. As consequence, diagnostic procedures must offer good sensitivity, but also high negative predictive value. US criteria distinguishing benign from metastatic or suspicious LNs have been described but they lack accuracy [4]. US-guided fine needle cytology (FNAC) proved to be a reliable method in examining the neck in patients who were previously treated for thyroid cancer $[5,6]$. However, sensitivity of FNAC is far from excellent, varying from $75 \%$ to $85 \%$, and altered by high rate of nondiagnostic or false-negative samples. To improve the diagnostic yield of FNAC, several authors have proposed measurement of $\mathrm{Tg}$ in aspirates (FNAC-Tg), particularly in the cases involving small, partially cystic, lymph nodes [7-12]. On the basis of prior studies, an increased $\mathrm{Tg}$ level in the needle washout has been shown to directly reflect the status of metastatic lymph nodes in patients affected by differentiated thyroid carcinoma. However, controversies still persist concerning some issues. First, there are few studies to validate the benefit of FNAC-Tg over FNAC alone, and, second, cut-off values ranging from 0.9 to $39 \mathrm{ng} / \mathrm{mL}$ have been suggested for FNAC$\mathrm{Tg}$, depending on the method used for the measurement $[7,13-15]$. As consequence, the exact place for FNACTg in the management of DTC patients is still debated. 
This study was then undertaken to (1) determine a diagnostic cut-off value for washout $\mathrm{Tg}$ in patients treated by total thyroidectomy for detecting recurrences, and (2) to compare the performance of the Tg cut-off value to US-guided FNAC for detecting DTC recurrences.

\section{Materials and Methods}

Our institutional review board approved our research study, and all subjects gave written informed consent.

2.1. Patients. Between January 2006 and February 2009 a total of 126 consecutive US-guided FNACs were performed on enlarged LNs. The samples were obtained from 108 patients (19 males, 89 females; age $42.7 \pm 18.2$ years; 91 patients with 1,94 with 2 , and 5 with 3 lesions, resp.). All patients had histologically confirmed primary DTC (papillary, $n=99$, including two tall-cell variants; follicular, $n=9$, including 2 Hürtle cells carcinomas). The primitive carcinoma was classified pT1 in 34 cases, pT2 in 43 cases, pT3 in 24 cases, and pT4 in 7 cases. Forty-five patients (42\%) had LN metastases at diagnosis. All patients underwent (near) total thyroidectomy and subsequent radioiodine ablation (administered activity from 1.85 to $3.70 \mathrm{GBq}$ ). The US criteria for possible malignant infiltration of lymph nodes were rounded contour, irregular internal echogenicity, punctate calcifications, fluids components, and abnormal colour Doppler pattern. Patients with positive cytology and/or FNAC-Tg measurement $(n=86,96$ lesions) underwent surgery and, if necessary, further radioiodine treatments. The diagnosis was confirmed in all cases by surgical pathology examination. Patients with negative FNAC-Tg measurement and cytology ( $n=22$, 30 lesions) underwent further follow up by serial clinical examinations, serum Tg measurements, neck US, and, whenever necessary, additional imaging procedures (i.e., radioiodine scan, ${ }^{18}$ FDG-PET/CT). No DTC recurrence was detected among these patients (follow up: mean 36 months, range 15-42 months).

2.2. US-Guided FNAC Procedure. All US-guided FNAC procedures were performed on supine patients with the neck hyperextended under continuous real-time US guidance with a high-resolution transducer (ACUSON $\times 150$, Siemens, Erlangen, Germany). Each lesion was aspirated at least twice by a $21 \mathrm{G}$ needle. The needle was inserted obliquely within the transducer plane of view, and moved back and forth through the nodule to compensate for patient movement and needle deflection. Gradual aspiration was applied by a $20 \mathrm{~mL}$ syringe connected to Cameco's device. Contents of needles were expelled onto glass slides and smeared with a second slide to spread fluid across the surface. Slides were fixed in $95 \%$ ethanol, papanicolaou stained to identify cellular details, and read by our cytopathologist. Following collection of cytology samples the needles were washed by $1 \mathrm{~mL}$ of normal saline in a plain serum tube (Vacutainer Systems, Plymouth, UK) and the washout directly sent to the laboratory [16]. Cytological examinations were performed by experienced cytopathologists and expressed as (1) positive: presence of epithelial cells with atypical cytological characteristics, or with cytological features of papillary carcinoma; (2) negative: reactive lymphadenitis and absence of malignant cells; (3) inadequate or nondiagnostic: absence of cells or presence of blood cells.

2.3. $\mathrm{Tg}$ Measurement. Thyroglobulin was measured in fineneedle washouts using an immunoradiometric assay (IRMA) based on coated tubes with monoclonal antibodies directed against distinct epitopes of the molecule of Tg (DYNO test Tg-plus, BRAHMS Diagnostic GmbH, Berlin, Germany). With this measurement, analytic sensitivity, defined as the detectable minimum concentration different from zero (mean value +2 standard deviation), and functional sensitivity, defined as the lowest value that was measured with the precision of a maximum $20 \%$ interassay variance, were $0.08 \mathrm{ng} / \mathrm{mL}$ and $0.2 \mathrm{ng} / \mathrm{mL}$, respectively. We did not measure $\mathrm{Tg}$ antibodies (FNAC-TgAb) because the clinical performance of FNAC-Tg is unaffected by serum TgAb [17].

2.4. Data Analysis. Diagnostic performance (i.e., sensitivity, specificity, positive predictive value, negative predictive value, and accuracy) of FNAC and FNAC-Tg was evaluated by comparing the results of the two procedures to the status of the patients defined as follows: malignant lymph node from thyroid cancer was proved by histological examination of surgically resected LNs; benign lymph node was proved by negative histological examination of surgically respected LNs, or if disappearance or absence of evolution on imaging modalities was demonstrated at 12 months or more follow up.

The Chi-square $\left(\chi^{2}\right)$ test, performed with SAS version 9.1 for Windows (SAS Institute, Cary, NC, US) was employed to compare the diagnostic rate of FNAC and FNAC-Tg. The FNAC-Tg receiver operating characteristic curve was developed using MedCalc 6.1 software (MedCalc Software, Mariakerke, Belgium). The cut-off values which maximise the sum of sensitivity plus specificity were determined as the points in the upper left hand corner. A $P$ value $<.05$ was considered to indicate statistical significance.

\section{Results}

Patients characteristics and cytological, pathological, and biochemical data are displayed in Table 1. Of the 126 lymph node lesions assessed for postoperative recurrences by US-guided FNAC and FNAC-Tg, 86 (68\%) lesions were finally diagnosed as malignant and the remaining 40 (32\%) lesions were diagnosed as benign LNs, respectively. The final diagnosis of the 86 malignant and 8 benign LN was established by surgical pathology; the remaining 32 benign lesions were diagnosed based on imaging follow up after at least 1 year. The time from thyroid ablation to US-FNAC was $19.5 \pm 14.31$ and $19.4 \pm 13.76$ months in patients with benign and malignant lesions, respectively ( $P$ not significant). Serum Tg levels were higher in patients with malignant lesions (median $4.20 \mathrm{ng} / \mathrm{mL}$, range $<0.2-27.10 \mathrm{ng} / \mathrm{mL}$ ) than those with benign lesions (median $.80 \mathrm{ng} / \mathrm{mL}$, range $<0.2-$ $2.70 \mathrm{ng} / \mathrm{mL} ; P<.0001)$. Serum TgAb were positive in 11 
TABLe 1: Patients characteristics and cytological, pathological, and biochemical data.

\begin{tabular}{|c|c|c|c|c|c|c|c|c|c|c|}
\hline \multirow{2}{*}{ Patients } & \multirow{2}{*}{ Histology } & \multirow{2}{*}{ pTNM } & \multirow{2}{*}{$\begin{array}{l}\text { Duration } \\
\text { (months) }\end{array}$} & \multirow{2}{*}{$\begin{array}{c}\mathrm{Tg} \\
(\mathrm{ng} / \mathrm{mL})\end{array}$} & \multirow{2}{*}{$\begin{array}{c}\text { TgAb } \\
(\mathrm{IU} / \mathrm{mL})\end{array}$} & \multirow{2}{*}{ Lesions } & \multirow{2}{*}{ Sites } & \multicolumn{2}{|c|}{ FNA } & \multirow{2}{*}{$\begin{array}{l}\text { Final } \\
\text { diagnosis }\end{array}$} \\
\hline & & & & & & & & Cytology & $\begin{array}{c}\mathrm{Tg} \\
(\mathrm{ng} / \mathrm{mL})\end{array}$ & \\
\hline 1 & PTC & pT1N1Mx & 6 & 1.0 & $<60$ & 1 & R II & ND & $<0.2$ & B \\
\hline 2 & PTC & pT1NxMx & 15 & 4.6 & $<60$ & 1 & L III & Negative & 435.2 & M \\
\hline 3 & PTC & pT1NxMx & 18 & 1.7 & $<60$ & 1 & R IV & CTM & 85.3 & M \\
\hline 4 & PTC & pT1NxMx & 30 & 0.4 & $<60$ & 1 & R III & ND & $<0.2$ & B \\
\hline 5 & PTC & pT1N0Mx & 6 & 3.1 & $<60$ & 1 & L II & ND & 97.2 & $\mathrm{M}$ \\
\hline 6 & PTC & pT2N0Mx & 19 & 9.1 & $<60$ & 2 & R IV, VI & CTM & 118.5 & M \\
\hline 7 & PTC & pT2NxMx & 47 & $<0.2$ & 334 & 1 & L IV & СТM & 879.4 & M \\
\hline 8 & PTC & pT2N1Mx & 6 & 0.9 & 78 & 1 & VI & Negative & 1.1 & B \\
\hline 9 & PTC & pT1N1Mx & 9 & 9.8 & $<60$ & 1 & L II & ND & 1348.6 & M \\
\hline 10 & PTC & pT2N0Mx & 14 & 1.5 & $<60$ & 1 & L III & CTM & 358.5 & M \\
\hline 11 & PTC & pT1NxMx & 8 & 12.6 & $<60$ & 3 & R II & CTM & 2387.4 & $\mathrm{M}$ \\
\hline 12 & PTC & pT2NxMx & 16 & 9.7 & $<60$ & 1 & R III & CTM & 875.9 & $\mathrm{M}$ \\
\hline 13 & PTC & pT1NxMx & 9 & 6.3 & $<60$ & 1 & R II & CTM & 958.6 & M \\
\hline 14 & PTC & pT1N0Mx & 11 & 1.0 & $<60$ & 1 & L III & Negative & 48.7 & M \\
\hline 15 & PTC & $\mathrm{pT} 2 \mathrm{~N} 1 \mathrm{Mx}$ & 39 & 9.5 & $<60$ & 1 & L IV & CTM & $>3000$ & $\mathrm{M}$ \\
\hline 16 & PTC & pT1NxMx & 12 & 0.8 & $<60$ & 1 & L III & CTM & 107.5 & M \\
\hline 17 & PTC & pT2NxMx & 19 & 2.7 & $<60$ & 3 & R III-IV & $\mathrm{ND}, \mathrm{CTM}$ & 541.6 & $\begin{array}{c}\text { RIII B, IV } \\
\mathrm{M}\end{array}$ \\
\hline 18 & PTC & pT4N1Mx & 6 & 1.6 & $<60$ & 1 & L III & CTM & 31.8 & M \\
\hline 19 & PTC & pT1N1Mx & 15 & 0.5 & 289 & 1 & R IV & ND & $<0.2$ & $\mathrm{~B}$ \\
\hline 20 & PTC & pT2N1Mx & 3 & 2.3 & $<60$ & 1 & L III & ND & 540.7 & M \\
\hline 21 & PTC & pT1NxMx & 34 & 4.1 & $<60$ & 1 & L III & СТM & 650.6 & M \\
\hline 22 & PTC & pT2N1Mx & 8 & $<0.2$ & 98 & 1 & L II & ND & 5.8 & $\mathrm{M}$ \\
\hline 23 & PTC & pT1NxMx & 9 & 0.8 & $<60$ & 2 & R II, R III & CTM & 87.5 & $\mathrm{M}$ \\
\hline 24 & PTC & pT4N0Mx & 10 & 1.4 & 755 & 1 & R II & CTM & 196.4 & $\mathrm{M}$ \\
\hline 25 & PTC & pT2NxMx & 21 & 11 & $<60$ & 1 & R III & ND & 585.3 & $\mathrm{M}$ \\
\hline 26 & PTC & pT1NxMx & 9 & $<0.2$ & $<60$ & 1 & R III & Negative & $<0.2$ & B \\
\hline 27 & PTC & pT4N1M & 12 & 1.1 & 160 & 1 & L III & Negative & 0.3 & B \\
\hline 28 & PTC & pT1N0Mx & 23 & 5.3 & $<60$ & 1 & R III & СТM & 784.2 & $\mathrm{M}$ \\
\hline 29 & PTC & pT2N1Mx & 5 & $<0.2$ & $>1000$ & 1 & R III & ND & $<0.2$ & $\mathrm{~B}$ \\
\hline 30 & PTC & pT2N0Mx & 22 & 4.9 & $<60$ & 1 & R III & CTM & 641.5 & $\mathrm{M}$ \\
\hline 31 & PTC & pT1NxMx & 36 & 0.3 & 367 & 1 & R IV & Negative & $<0.2$ & $\mathrm{~B}$ \\
\hline 32 & PTC & pT1NxMx & 15 & 7.1 & $<60$ & 1 & R III & ND & 665.4 & $\mathrm{M}$ \\
\hline 33 & PTC & pT2N0Mx & 41 & $<0.2$ & $<60$ & 1 & L II & Negative & $<0.2$ & B \\
\hline 34 & PTC & pT1NxMx & 24 & 2.6 & $<60$ & 1 & R III & CTM & 570.6 & $\mathrm{M}$ \\
\hline 35 & PTC & pT4N0Mx & 31 & 1.4 & $>1000$ & 3 & L IV, VI & СТM & 96.7 & $\mathrm{M}$ \\
\hline 36 & PTC & pT4N1M & 12 & 27.1 & $<60$ & 1 & R II & CTM & 2987.3 & $\mathrm{M}$ \\
\hline 37 & PTC & pT1N1Mx & 54 & 6.8 & $<60$ & 1 & L III & CTM & 875.6 & $\mathrm{M}$ \\
\hline 38 & PTC & pT2NxMx & 12 & $<0.2$ & 149 & 1 & R IV & Negative & $<0.2$ & $\mathrm{~B}$ \\
\hline 39 & PTC & pT2N0Mx & 9 & 0.9 & $<60$ & 1 & R II & Negative & $<0.2$ & $\mathrm{~B}$ \\
\hline 40 & PTC & pT1N0Mx & 34 & 2.4 & 89 & 1 & L III & ND & 347.4 & $\mathrm{M}$ \\
\hline 41 & PTC & pT2NxMx & 14 & 5.1 & $<60$ & 1 & L IV & ND & 1655.7 & $\mathrm{M}$ \\
\hline 42 & PTC & pT4N1M & 8 & 7.7 & $<60$ & 1 & L III & Negative & 2076.5 & $\mathrm{M}$ \\
\hline 43 & PTC & pT1NxMx & 61 & 1.1 & $<60$ & 1 & VI & Negative & $<0.2$ & B \\
\hline 44 & PTC & pT2N0Mx & 22 & 1.0 & $<60$ & 1 & L II & СТM & 90.6 & $\mathrm{M}$ \\
\hline
\end{tabular}


Table 1: Continued.

\begin{tabular}{|c|c|c|c|c|c|c|c|c|c|c|}
\hline \multirow{2}{*}{ Patients } & \multirow{2}{*}{ Histology } & \multirow{2}{*}{ pTNM } & \multirow{2}{*}{$\begin{array}{l}\text { Duration } \\
\text { (months) }\end{array}$} & \multirow{2}{*}{$\begin{array}{c}\mathrm{Tg} \\
(\mathrm{ng} / \mathrm{mL})\end{array}$} & \multirow{2}{*}{$\begin{array}{c}\text { TgAb } \\
(\mathrm{IU} / \mathrm{mL})\end{array}$} & \multirow{2}{*}{ Lesions } & \multirow{2}{*}{ Sites } & \multicolumn{2}{|c|}{ FNA } & \multirow{2}{*}{$\begin{array}{c}\text { Final } \\
\text { diagnosis }\end{array}$} \\
\hline & & & & & & & & Cytology & $\begin{array}{c}\mathrm{Tg} \\
(\mathrm{ng} / \mathrm{mL})\end{array}$ & \\
\hline 45 & PTC & pT2N1Mx & 12 & 6.4 & $<60$ & 2 & R III-IV & CTM, ND & 458.6 & $\begin{array}{c}\text { RIII M, IV } \\
\text { B }\end{array}$ \\
\hline 46 & PTC & pT1NxMx & 45 & 0.6 & $<60$ & 1 & R IV & Negative & $<0.2$ & B \\
\hline 47 & PTC & pT4N0Mx & 36 & 4.3 & $<60$ & 1 & R III & CTM & 766.4 & M \\
\hline 48 & PTC & pT2NxMx & 11 & 1.9 & $<60$ & 1 & L II & ND & 306.5 & M \\
\hline 49 & PTC & pT3NxMx & 18 & 7.0 & $<60$ & 1 & R III & CTM & 955.6 & M \\
\hline 50 & PTC & pT2NxMx & 10 & 1.2 & $<60$ & 1 & L III & Negative & $<0.2$ & $\mathrm{~B}$ \\
\hline 51 & PTC & pT1N1Mx & 12 & 3.9 & $<60$ & 1 & R III & CTM & 564.7 & M \\
\hline 52 & PTC & pT3N0Mx & 25 & 6.4 & 190 & 1 & R III & ND & 1078.6 & M \\
\hline 53 & PTC & pT2N1Mx & 16 & 9.5 & $<60$ & 1 & L IV & CTM & 759.4 & M \\
\hline 54 & PTC & pT1NxMx & 9 & 0.6 & $<60$ & 1 & R III & Negative & $<0.2$ & B \\
\hline 55 & PTC & pT3N1Mx & 60 & $<0.2$ & 107 & 2 & L II-III & CTM & 137.5 & M \\
\hline 56 & PTC & pT1N0Mx & 11 & 5.3 & $<60$ & 1 & R II & CTM & 654.8 & M \\
\hline 57 & PTC & pT2N1Mx & 18 & 1.1 & 860 & 1 & L III & ND & $<0.2$ & B \\
\hline 58 & PTC & pT1NxMx & 9 & 0.9 & $<60$ & 1 & R IV & Negative & $<0.2$ & B \\
\hline 59 & PTC & pT2NxMx & 28 & 1.1 & $<60$ & 1 & R III & ND & 99.6 & M \\
\hline 60 & PTC-TCV & pT3NxMx & 13 & 4.6 & $<60$ & 1 & L II & CTM & 436.8 & M \\
\hline 61 & PTC & pT1NxMx & 2 & 2.7 & $<60$ & 1 & R II & CTM & 194.5 & $\mathrm{M}$ \\
\hline 62 & PTC & pT3NxMx & 7 & 21.9 & $<60$ & 3 & R III, L II & $\begin{array}{l}\text { Neg., } \\
\text { CTM }\end{array}$ & $>3000$ & $\begin{array}{c}\text { RIII B, LII } \\
M\end{array}$ \\
\hline 63 & PTC & pT1NxMx & 18 & 1.1 & $<60$ & 1 & R III & CTM & 27.4 & M \\
\hline 64 & PTC & pT3NxMx & 10 & 3.5 & $<60$ & 1 & VI & CTM & 147.4 & M \\
\hline 65 & PTC & pT2N0Mx & 29 & $<0.2$ & $>1000$ & 1 & R IV & Negative & $<0.2$ & B \\
\hline 66 & PTC & pT3N1Mx & 6 & 5.6 & $<60$ & 1 & L III & CTM & $<0.2$ & M \\
\hline 67 & PTC & pT1N1Mx & 11 & 2.1 & $<60$ & 1 & R III & ND & $<0.2$ & M \\
\hline 68 & PTC & pT3N0Mx & 18 & 0.7 & $<60$ & 1 & R III & ND & $<0.2$ & B \\
\hline 69 & PTC & pT2N0Mx & 23 & 1.7 & $<60$ & 1 & L IV & CTM & 75.6 & M \\
\hline 70 & PTC & pT1NxMx & 9 & 3.5 & $<60$ & 1 & L II & ND & 72.6 & $\mathrm{M}$ \\
\hline 71 & PTC & pT3N0Mx & 18 & 7.1 & $<60$ & 2 & R IV, VI & CTM & 386.2 & $\begin{array}{c}\text { RIV B, VI } \\
M\end{array}$ \\
\hline 72 & PTC & pT1NxMx & 7 & 1.8 & & 1 & L II & Negative & $<0.2$ & B \\
\hline 73 & PTC & pT2NxMx & 11 & 3.6 & $<60$ & 1 & R III & ND & 245.7 & $\mathrm{M}$ \\
\hline 74 & PTC & pT2NxMx & 29 & 2.2 & $<60$ & 2 & L III-IV & CTM & 67.4 & IV M, III B \\
\hline 75 & PTC & pT2N1Mx & 14 & 5.7 & $<60$ & 1 & L II & CTM & 198.7 & M \\
\hline 76 & PTC & pT1N0Mx & 8 & 1.5 & $<60$ & 1 & R IV & CTM & 26.1 & M \\
\hline 77 & PTC & pT2NxMx & 10 & 2.1 & $<60$ & 1 & VI & CTM & 59.7 & M \\
\hline 78 & PTC & pT1NxMx & 18 & 0.7 & $<60$ & 1 & R IV & Negative & $<0.2$ & B \\
\hline 79 & PTC & pT3N1Mx & 34 & 12.3 & $<60$ & 1 & R III & ND & 678.4 & M \\
\hline 80 & PTC & pT1N1Mx & 16 & 1.8 & $<60$ & 1 & L II & Negative & $<0.2$ & B \\
\hline 81 & PTC & pT2N0Mx & 52 & 0.9 & $<60$ & 1 & R III & Negative & $<0.2$ & B \\
\hline 82 & PTC & pT2N1Mx & 25 & 9.4 & $<60$ & 1 & L III & ND & 563.6 & M \\
\hline 83 & PTC & pT1NxMx & 9 & 4.1 & $<60$ & 1 & VI & CTM & 64.7 & M \\
\hline 84 & PTC & pT2N1Mx & 18 & 3.1 & $<60$ & 1 & R IV & CTM & 116.4 & M \\
\hline 85 & PTC-TCV & pT2NxMx & 20 & 15.5 & $<60$ & 3 & R IV-L III & CTM & 432.9 & M \\
\hline 86 & PTC & pT3N0Mx & 8 & $<0.2$ & 56 & 1 & VI & ND & $<0.2$ & B \\
\hline 87 & PTC & pT1N0Mx & 31 & 2.9 & $<60$ & 1 & R IV & CTM & 39.3 & M \\
\hline 88 & PTC & pT2NxMx & 11 & 1.7 & $<60$ & 1 & R III & Negative & $<0.2$ & B \\
\hline 89 & PTC & pT2NxMx & 54 & 6.5 & $<60$ & 1 & L IV & ND & 467.7 & M \\
\hline 90 & PTC & pT1N1Mx & 28 & 0.3 & 651 & 1 & R II & Negative & $<0.2$ & B \\
\hline 91 & PTC & pT2NxMx & 12 & 2.1 & $<60$ & 1 & L III & CTM & 88.6 & M \\
\hline
\end{tabular}


TABle 1: Continued.

\begin{tabular}{|c|c|c|c|c|c|c|c|c|c|c|}
\hline \multirow{2}{*}{ Patients } & \multirow{2}{*}{ Histology } & \multirow{2}{*}{ pTNM } & \multirow{2}{*}{$\begin{array}{l}\text { Duration } \\
\text { (months) }\end{array}$} & \multirow{2}{*}{$\begin{array}{c}\mathrm{Tg} \\
(\mathrm{ng} / \mathrm{mL})\end{array}$} & \multirow{2}{*}{$\begin{array}{c}\text { TgAb } \\
(\mathrm{IU} / \mathrm{mL})\end{array}$} & \multirow{2}{*}{ Lesions } & \multirow{2}{*}{ Sites } & \multicolumn{2}{|c|}{ FNA } & \multirow{2}{*}{$\begin{array}{l}\text { Final } \\
\text { diagnosis }\end{array}$} \\
\hline & & & & & & & & Cytology & $\begin{array}{c}\mathrm{Tg} \\
(\mathrm{ng} / \mathrm{mL})\end{array}$ & \\
\hline 92 & PTC & pT3N0Mx & 17 & 9.4 & $<60$ & 2 & R IV, VI & CTM & 156.2 & IV M, VI B \\
\hline 93 & PTC & pT1NxMx & 22 & 1.0 & $<60$ & 1 & L IV & Negative & $<0.2$ & B \\
\hline 94 & PTC & pT2N1Mx & 9 & 4.3 & $<60$ & 1 & VI & Negative & 53.8 & M \\
\hline 95 & PTC & pT2N0Mx & 26 & $<0.2$ & $<60$ & 1 & R IV & Negative & $<0.2$ & $\mathrm{~B}$ \\
\hline 96 & PTC & pT1N0Mx & 12 & 0.9 & $<60$ & 1 & R III & ND & 2.7 & M \\
\hline 97 & PTC & pT2NxMx & 17 & 8.1 & $<60$ & 1 & L II & ND & 356.4 & M \\
\hline 98 & PTC & pT2NxMx & 9 & 0.9 & $<60$ & 1 & R III & CTM & 19.6 & M \\
\hline 99 & PTC & pT3N1Mx & 12 & 1.1 & $<60$ & 1 & L III & ND & 0.6 & $\mathrm{~B}$ \\
\hline 100 & FTC & pT1N1Mx & 45 & 4.9 & $<60$ & 1 & R III & CTM & 117.5 & M \\
\hline 101 & FTC & pT3N0Mx & 36 & 1.4 & $<60$ & 1 & R III & Negative & $<0.2$ & B \\
\hline 102 & FTC & pT2N1Mx & 11 & $<0.2$ & $>1000$ & 2 & R III-IV & Negative & $<0.2$ & B \\
\hline 103 & FTC (HC) & pT1NxMx & 72 & 10.4 & $<60$ & 1 & R IV & ND & 278.9 & M \\
\hline 104 & FTC & pT2N1Mx & 11 & 0.7 & $<60$ & 1 & R III & Negative & $<0.2$ & B \\
\hline 105 & FTC & pT1N0Mx & 18 & 2.1 & $<60$ & 1 & R IV & СТM & 74.4 & M \\
\hline 106 & FTC (HC) & pT1N0Mx & 6 & 1.9 & $<60$ & 1 & L III & Negative & 0.8 & B \\
\hline 107 & FTC & pT2N1Mx & 25 & 7.8 & $<60$ & 1 & R II & ND & 116.4 & M \\
\hline 108 & FTC & pT1NxMx & 19 & 4.6 & $<60$ & 2 & L II-III & CTM & 89.5 & M \\
\hline
\end{tabular}

FNA, fine-needle aspiration; PTC, papillary thyroid carcinoma; FTC, follicular thyroid carcinoma; TCV, tall-cell variant; HC Hürtle cell; R, right, L, left; duration, time from thyroid ablation to FNA; II-III-IV, upper, middle, lower neck lateral compartment, IV, central neck compartment.

TABLE 2: Diagnostic performance of FNAC cytology as compared to final diagnosis.

\begin{tabular}{|c|c|c|}
\hline \multirow[b]{2}{*}{ FNAC } & \multicolumn{2}{|c|}{ Final diagnosis } \\
\hline & $\begin{array}{l}\text { Malignant LNs } \\
\quad(n=86)\end{array}$ & $\begin{array}{c}\text { Benign LNs } \\
\quad(n=40)\end{array}$ \\
\hline Positive & 61 & 0 \\
\hline Negative & 4 & 32 \\
\hline Inadequate & 21 & 8 \\
\hline
\end{tabular}

TABLE 3: Diagnostic performance of FNAC-Tg as compared to final diagnosis.

\begin{tabular}{lcc}
\hline & $\begin{array}{c}\text { Malignant } \\
\text { LNs }(n=86)\end{array}$ & $\begin{array}{c}\text { Benign LNs } \\
(n=40)\end{array}$ \\
\hline FNAC-Tg $>1.1 \mathrm{ng} / \mathrm{mL}$ & 86 & 0 \\
FNAC-Tg $\leq 1.1 \mathrm{ng} / \mathrm{mL}$ & 0 & 40 \\
\hline
\end{tabular}

TABLE 4: Figures of merits of FNAC cytology and FNAC Tg.

\begin{tabular}{lccccc}
\hline FNAC & Sensitivity & Specificity & PPV & NPV & Accuracy \\
\hline Cytology & $71 \%$ & $80 \%$ & $88 \%$ & $56 \%$ & $74 \%$ \\
Tg & $100 \%$ & $100 \%$ & $100 \%$ & $100 \%$ & $100 \%$ \\
\hline
\end{tabular}

of 40 patients with benign lesions and 7 of 67 patients with malignant lesions $(P<.001)$. The rate of FNAC samples adequate for cytological examination was $77 \%$ (97 samples) in contrast FNAC-Tg available in 100\% of aspirates $(P<.01)$. As shown in Table 2 cytological examination correctly identified 61 malignant LNs, was negative in 4 , and
TABLE 5: Rate of positive FNAC-Tg values (i.e., $>1.1 \mathrm{ng} / \mathrm{mL}$ ) in patients with inadequate or misdiagnosed FNAC-cytology.

\begin{tabular}{lcc}
\hline Final status & $\begin{array}{c}\text { FNAC inadequate } \\
(n=29)\end{array}$ & $\begin{array}{c}\text { FNAC } \\
\text { false-negative } \\
(n=4)\end{array}$ \\
\hline $\begin{array}{l}\text { Malignant LNs } \\
(n=25)\end{array}$ & $21 / 21$ & $4 / 4$ \\
$\begin{array}{l}\text { Benign LNs } \\
(n=8)\end{array}$ & $0 / 8$ & - \\
\hline
\end{tabular}

inadequate in 21. For benign LNs, FNAC was negative in 32 and inadequate in 8. It showed no false-positive results. Thus, sensitivity, specificity, positive predictive value (PPV), negative predictive value (NPV), and accuracy of FNAC were $71 \%, 80 \%, 88 \%, 56 \%$, and $74 \%$, respectively. The ROC curve analysis demonstrated that the most appropriate cut-off value for the diagnosis of thyroid cancer metastatic lesions was $1.1 \mathrm{ng} / \mathrm{mL}$ (sensitivity $100 \%$, specificity $100 \%$, PPV 100\%, NPV 100\%, accuracy 100\%; Figure 1, Tables 3, and 4). Basing on this cut-off level, the FNAC-Tg results correctly concluded all 25 malignant (100\%) and 8 benign $(100 \%)$ cases with false-negative $(n=4)$ and nondiagnostic $(n=29)$ FNAC results, respectively (Table 5$)$. The FNAC$\mathrm{Tg}$ levels were significantly higher in malignant (median $513.8 \mathrm{ng} / \mathrm{mL}$, range $1.7->3000 \mathrm{ng} / \mathrm{mL}$ ) than benign (median $<0.2 \mathrm{ng} / \mathrm{mL}$, range $<0.2-1.1 \mathrm{ng} / \mathrm{mL})$ lesions, respectively $(P<$ $.0000001)$. Particularly, specimen Tg levels were undetectable (i.e., $<0.2 \mathrm{ng} / \mathrm{mL}$ ) in 36 cases and were $0.3,0.6,0.8$, and $1.1 \mathrm{ng} / \mathrm{mL}$ in remaining 4 cases with benign lesions. 


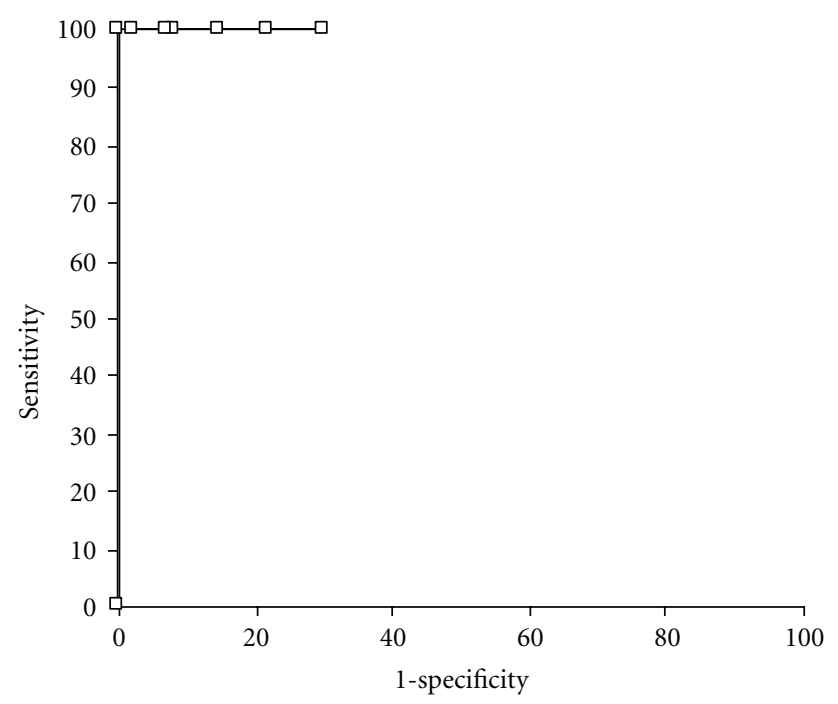

FIgURE 1: FNAC-Tg: ROC curve analysis.

\section{Discussion}

An accurate discrimination between metastatic and reactive LNs is essential in the management of thyroid cancer. Cytological examination of FNAC samples disclosed by US has been the most accurate method to diagnose a cervical LN. However, as showed also in our study, its sensitivity is negatively impacted by the rate of nondiagnostic samples although FNAC procedures are performed by USexperienced physicians and dedicated cytopathologists $[5,6$, $15]$. In the present study $23 \%$ of samples were nondiagnostic; this perfectly conforms with previously reported data [18]. Cystic metastasis and partial LN involvement comprise most of the inadequate/nondiagnostic FNAC-cytology cases and could be misinterpreted as a benign cervical cystic mass or branchial cleft cysts and could therefore delay the correct diagnosis and a further radical neck lymphadenectomy. The immunocytochemical Tg staining on FNAC samples from of neck nodes was previously evaluated in patients with DTC. Because an adequate FNAC sample is required, however, the practical impact of this technique is limited in clinical practice [19]. Recently, the FNAC-Tg measurement has been proposed to be a useful diagnostic technique in the management of patients with thyroid cancer. Because $\mathrm{Tg}$ is produced only by follicular thyrocyte-derived cells, measurement of $\mathrm{Tg}$ in FNAC specimens of nonthyroidal tissues enables the detection of persistence, recurrence, or metastasis of differentiated thyroid carcinoma. In our study FNAC-Tg analysis was more sensitive for detecting metastasis when compared with FNAC alone, and allows the accurate diagnosis in samples with inconclusive cytology. Our results perfectly conforms those recently reported by Bournaud and colleagues [18]. By contrast Tg could be determined in all aspirates and a sensitivity of $100 \%$ was achieved in our series, that is at the higher end of previously reported data $(81 \%-100 \%)[7,9,14,17,18]$. Although the performance of FNAC-Tg is well established, the Tg threshold value remains controversial. The $\mathrm{Tg}$ assays employed and methods for determining the cut-off value differed from one study to another, resulting in a large range, from $0.9 \mathrm{ng} / \mathrm{mL}$ to values as high as $39 \mathrm{ng} / \mathrm{mL}$, proposed in the literature.

In our study the best $\mathrm{Tg}$ threshold was determined at $1.1 \mathrm{ng} / \mathrm{mL}$ by ROC curve analysis. Using a threshold of $1.1 \mathrm{ng} / \mathrm{mL}$ we observe neither false-positive results in nonmalignant LNs nor false-negative results in malignant LNs at final diagnosis. Additionally, FNAC-Tg results correctly classified as malignant 4 lesions that tested negatively in cytological examination. All in all, our results are in accordance with those of Snozek and colleagues that used a Tg assay with a functional sensitivity at $0.1 \mathrm{ng} / \mathrm{mL}$ and proposed a cut-off level of $1.00 \mathrm{ng} / \mathrm{mL}$ : basing on their results these authors suggested that FNAC-Tg should be substituted for FNAC in many cases [7]. Of importance, our samples were obtained in a population of well-differentiated thyroid carcinomas (i.e., only two Hürtle cell and two tall-cell variants among 108 DTC cases). Several authors reported, however, that FNAC-Tg levels could be undetectable in some types of thyroid cancers (i.e., poorly differentiated thyroid carcinomas) $[13,17]$. This correspond to the fact that amount and intensity of Tg expression parallel with differentiation of the tumor and could produce false-negative results. As a consequence, caution is needed, and a combination of FNAC and FNAC-Tg should remain the standard, especially in patients harboring less differentiated thyroid carcinomas.

\section{Conclusions}

The diagnostic performance of needle washout FNACTg measurement with a cut-off of $1.1 \mathrm{ng} / \mathrm{mL}$ compared favourably with cytology and allowed accurate diagnosis in all cases in whom cytology was nondiagnostic.

\section{Conflict of Interests}

The author report that there are no conflicts of interests.

\section{References}

[1] L. Davies and H. G. Welch, "Increasing incidence of thyroid cancer in the United States, 1973-2002," Journal of the American Medical Association, vol. 295, no. 18, pp. 2164-2167, 2006.

[2] M. J. Schlumberger, "Papillary and follicular thyroid carcinoma," The New England Journal of Medicine, vol. 338, no. 5, pp. 297-306, 1998.

[3] N. A. Johnson and M. E. Tublin, "Postoperative surveillance of differentiated thyroid carcinoma: rationale, techniques, and controversies," Radiology, vol. 249, no. 2, pp. 429-444, 2008.

[4] A. Frasoldati and R. Valcavi, "Challenges in neck ultrasonography: lymphadenopathy and parathyroid glands," Endocrine Practice, vol. 10, no. 3, pp. 261-268, 2004.

[5] M. O. Bernier, C. Moisan, G. Mansour, A. Aurengo, F. Ménégaux, and L. Leenhardt, "Usefulness of fine needle aspiration cytology in the diagnosis of loco-regional recurrence of differentiated thyroid carcinoma," European Journal of Surgical Oncology, vol. 31, no. 3, pp. 288-293, 2005.

[6] G. W. Boland, M. J. Lee, P. R. Mueller, W. Mayo-Smith, S. L. Dawson, and J. F. Simeone, "Efficacy of sonographically 
guided biopsy of thyroid masses and cervical lymph nodes," American Journal of Roentgenology, vol. 161, no. 5, pp. 10531056, 1993.

[7] C. L. H. Snozek, E. P. Chambers, C. C. Reading et al., "Serum thyroglobulin, high-resolution ultrasound, and lymph node thyroglobulin in diagnosis of differentiated thyroid carcinoma nodal metastases," Journal of Clinical Endocrinology and Metabolism, vol. 92, no. 11, pp. 4278-4281, 2007.

[8] S. J. Jeon, E. Kim, J. S. Park et al., "Diagnostic benefit of thyroglobulin measurement in fine-needle aspiration for diagnosing metastatic cervical lymph nodes from papillary thyroid cancer: correlations with US features," Korean Journal of Radiology, vol. 10, no. 2, pp. 106-111, 2009.

[9] M. J. Kim, E. K. Kim, B. M. Kim et al., "Thyroglobulin measurement in fine-needle aspirate washouts: the criteria for neck node dissection for patients with thyroid cancer," Clinical Endocrinology, vol. 70, no. 1, pp. 145-151, 2009.

[10] T. Uruno, A. Miyauchi, K. Shimizu et al., "Usefulness of thyroglobulin measurement in fine-needle aspiration biopsy specimens for diagnosing cervical lymph node metastasis in patients with papillary thyroid cancer," World Journal of Surgery, vol. 29, no. 4, pp. 483-485, 2005.

[11] Z. W. Baloch, J. E. Barroeta, J. Walsh et al., "Utility of thyroglobulin measurement in fine-needle aspiration biopsy specimens of lymph nodes in the diagnosis of recurrent thyroid carcinoma," CytoJournal, vol. 5, article 1, 2008.

[12] E. Sigstad, A. Heilo, E. Paus et al., "The usefulness of detecting thyroglobulin in fine-needle aspirates from patients with neck lesions using a sensitive thyroglobulin assay," Diagnostic Cytopathology, vol. 35, no. 12, pp. 761-767, 2007.

[13] A. L. Borel, R. Boizel, P. Faure et al., "Significance of low levels of thyroglobulin in fine needle aspirates from cervical lymph nodes of patients with a history of differentiated thyroid cancer," European Journal of Endocrinology, vol. 158, no. 5, pp. 691-698, 2008.

[14] N. Cunha, F. Rodrigues, F. Curado et al., "Thyroglobulin detection in fine-needle aspirates of cervical lymph nodes: a technique for the diagnosis of metastatic differentiated thyroid cancer," European Journal of Endocrinology, vol. 157, no. 1, pp. 101-107, 2007.

[15] A. Frasoldati, E. Toschi, M. Zini et al., "Role of thyroglobulin measurement in fine-needle aspiration biopsies of cervical lymph nodes in patients with differentiated thyroid cancer," Thyroid, vol. 9, no. 2, pp. 105-111, 1999.

[16] L. Giovanella, L. Ceriani, S. Suriano, and S. Crippa, "Thyroglobulin measurement on fine-needle washout fluids: influence of sample collection methods," Diagnostic Cytopathology, vol. 37, no. 1, pp. 42-44, 2009.

[17] F. Boi, G. Baghino, F. Atzeni, M. L. Lai, G. Faa, and S. Mariotti, "The diagnostic value for differentiated thyroid carcinoma metastases of thyroglobulin ( $\mathrm{Tg}$ ) measurement in washout fluid from fine-needle aspiration biopsy of neck lymph nodes is maintained in the presence of circulating anti-Tg antibodies," Journal of Clinical Endocrinology and Metabolism, vol. 91, no. 4, pp. 1364-1369, 2006.

[18] C. Bournaud, A. Charrié, C. Nozières et al., "Thyroglobulin measurement in fine-needle aspirates of lymph nodes in patients with differentiated thyroid cancer: a simple definition of the threshold value, with emphasis on potential pitfalls of the method," Clinical Chemistry and Laboratory Medicine, vol. 48, no. 8, pp. 1171-1177, 2010.

[19] T. Pisani, A. Vecchione, N. T. Sinopoli, A. Drusco, C. Valli, and M. R. Giovagnoli, "Cytological and immunocytochemical analysis of laterocervical lymph nodes in patients with previous thyroid carcinoma," Anticancer Research, vol. 19, no. 4, pp. 3527-3530, 1999. 


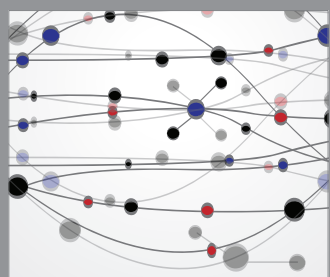

The Scientific World Journal
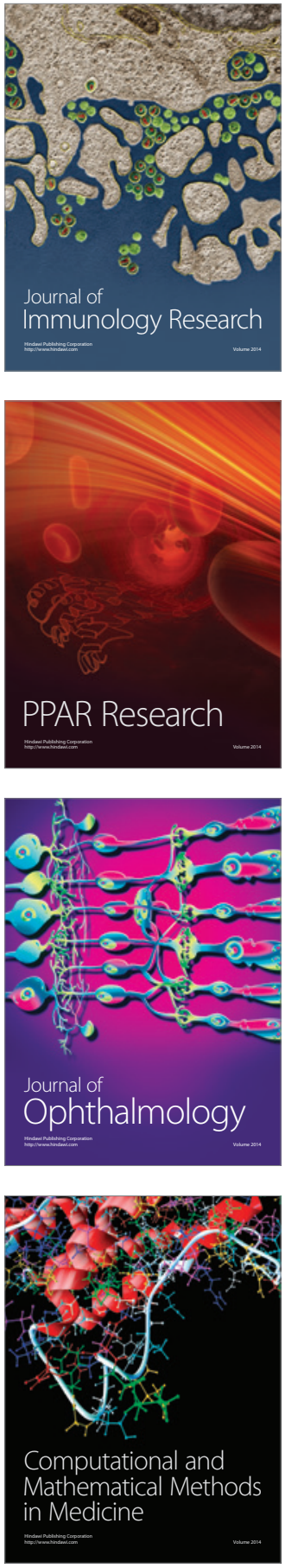

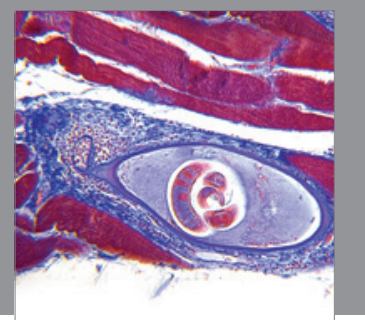

Gastroenterology

Research and Practice
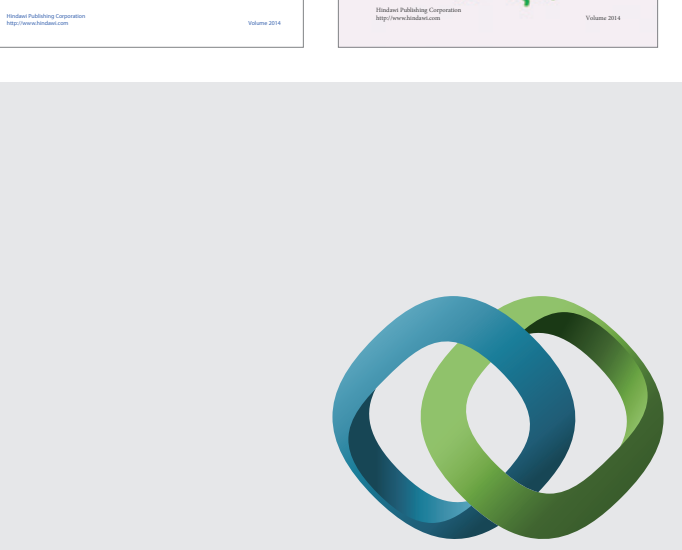

\section{Hindawi}

Submit your manuscripts at

http://www.hindawi.com
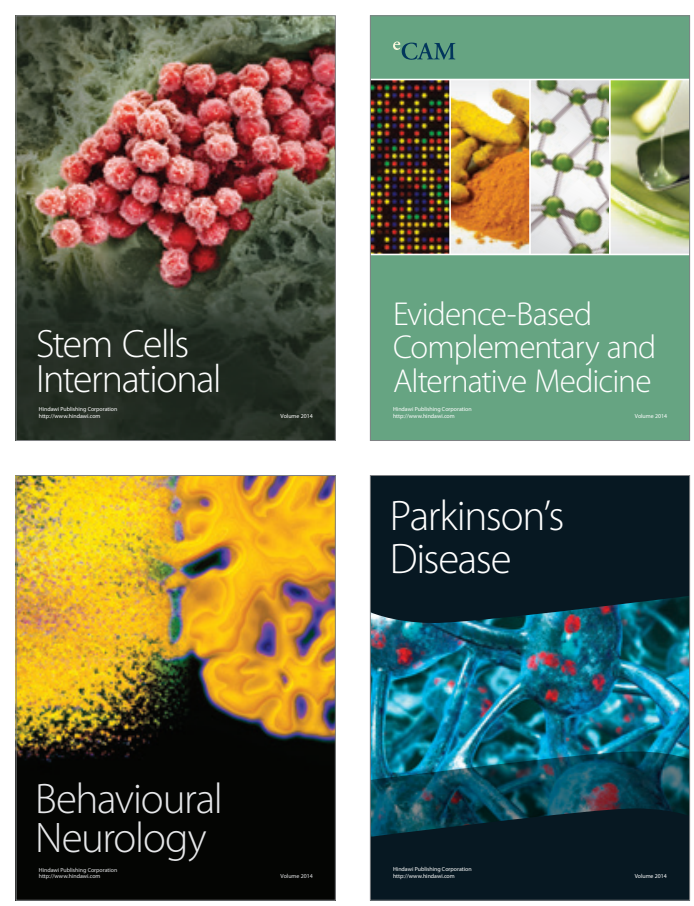

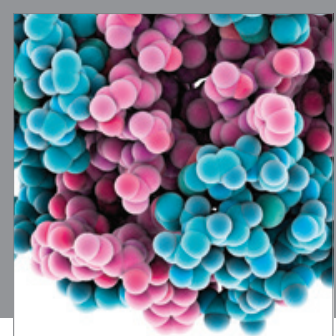

Journal of
Diabetes Research

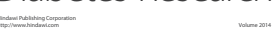

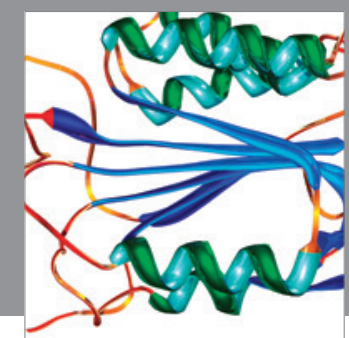

Disease Markers
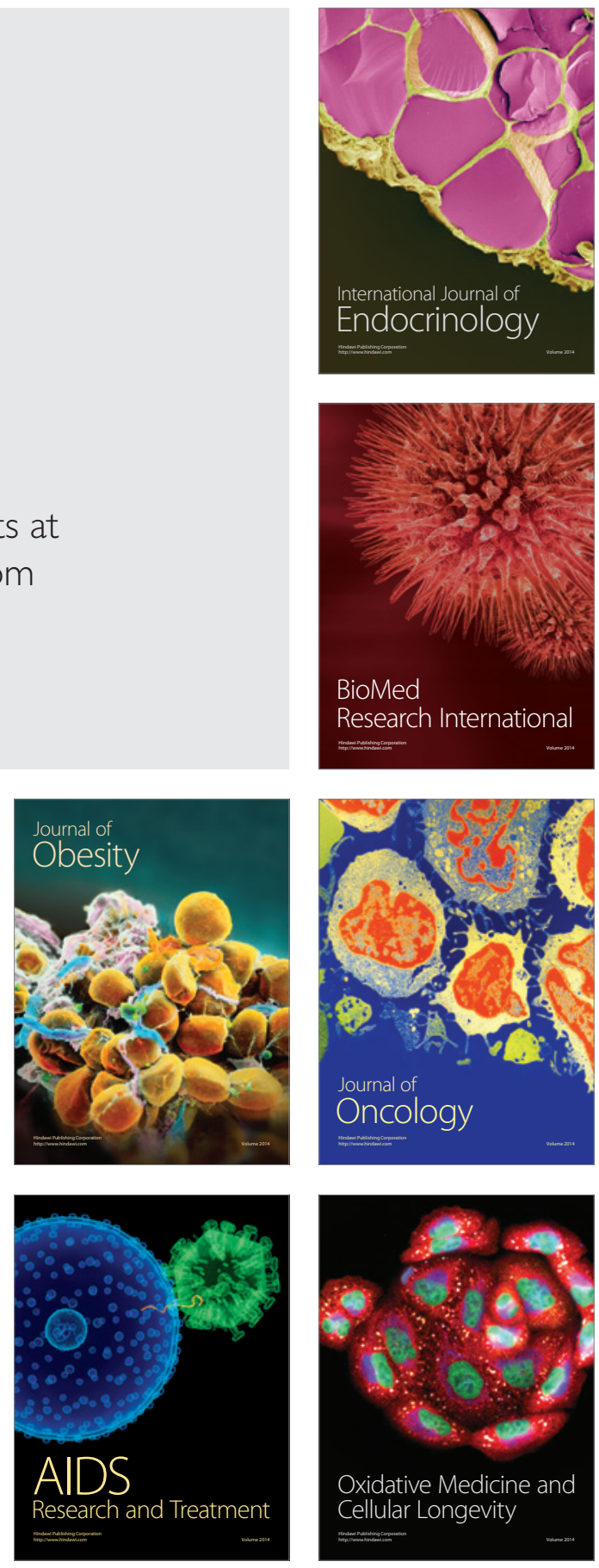